\title{
A genetic algorithm based train speed regulation optimization
}

\author{
C. Rongwu, Y. Tao \& B. R. Bisrat \\ School of Information Science and Technology, \\ Southwest Jiaotong University, China
}

\begin{abstract}
The intelligent train speed regulation algorithm plays a very important role in the automatic train control system. A genetic algorithm based time precision train speed regulation for the energy consumption optimization algorithm of a Communication Based Train Control System (CBTC) is studied in this paper. Compared to traditional performance level commands, which were generated by the Automatic Train Supervision (ATS) subsystem, the enhanced genetic algorithm based train speed regulation algorithm can be used to save energy consumption and improve passenger travel comfort at each defined inter-section of travel time. In this algorithm, each travel time target speed will be reflected by its particular speed profile, which has been optimized by the genetic algorithm with the object of energy saving. In the CBTC system, with the adoption of the bandwidth network of the train-wayside communication, this optimization process can be carried out offline or online in the ATS subsystem and transmit to the onboard Automatic Train Operation (ATO) subsystem to guide train operation control.
\end{abstract}

Keywords: genetic algorithm, time precision, energy saving, CBTC, train speed regulation, performance level.

\section{Introduction}

With the adoption of the moving block principle and the improvement of the system performance, CBTC has become the most important train control technique in the modern train control system. While among the techniques used in the CBTC system, train speed regulation and the related algorithm such as the energy consumption and the time precision operation need to be studied further. 
Jing et al. [1] and Rongwu and Jin [2] created the train operation model and set up a system simulation platform for the algorithm study. Yanbing et al. [3] studied the train traction energy consumption through the analysis of the train data and the track civil information. Ke and Chen [4] and Ruelland and Al-Haddad [5] provide the traction energy consumption saving strategy and the implementation for a different system. Jin [6], Jin et al. [9], Guohong [7] and Fei [8] studied the train operation optimization based on the multi-objects in the simulation system, which energy consumption saving as one of objects. Yusheng and Zhongsheng [10] and Bo [11] studied energy consumption based on the genetic algorithm.

In this paper the train control model and the train speed regulation principle were studied and based on the genetic algorithm, for the object of energy consumption saving and time precision train inter-station operation; the optimized train speed control profile was created during the simulation.

\section{CBTC train speed regulation}

\subsection{Train control model}

CBTC is a moving block system that adopted the IEEE standard braking model.

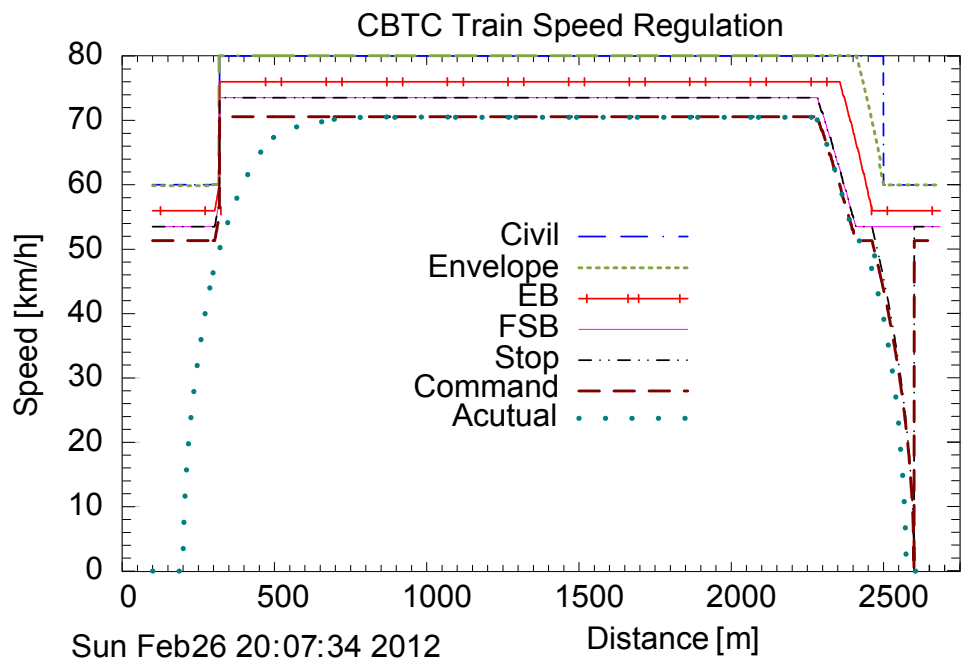

Figure 1: Train speed control model.

Fig. 1 is the application of the braking model for the train speed regulation used in the simulation system of this paper, in which civil speed is the not allowed exceeded speed in the worst case of the train. In this model, the emergency braking speed, full service braking speed and the command speed were all induced from the civil speed. Train will be controlled regarded to the command speed in the ATO system. 


\subsection{Performance level}

Performance Level is the train speed regulation command generated by ATS according to the time table and the actual train punctuality. In the current project design ATS simply calculates the train speed according to the maximum allow speed by reducing a certain percentage to form the corresponding performances level. In the simulation system of this paper the performance level 1, 2, 3, 4 and 5 (PL1, PL2, PL3, PL4 and PL5) were built by reducing 10\% from each higher speed level [13].

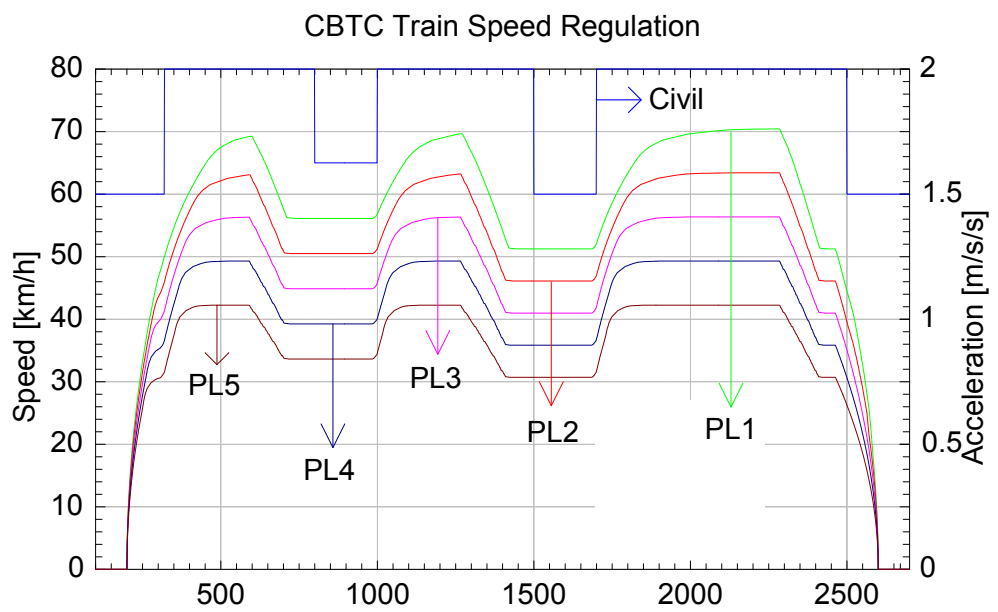

Sun Feb 26 20:20:12 2012 Distance [m]

Figure 2: Performance level.

There are 3 main problems in such performance level based train speed regulation:

i) Performance level based regulation is not a time precision control method. A train with the performance level command generated by ATS can only adjusted by limit number of speed profile;

ii) Performance level based regulation does not take the energy saving into consideration. In the practical civil situation there will always have the energy saving grade, see fig. 3. While with the performance level command, train can only be tracked at a constant acceleration when the train leaving a station. It is most likely that train has reached the maximum command speed when the train is still on the downgrade section, i.e. train must be braked to keep the train avoid hitting the braking speed profile. This situation will unavoidably cause the unnecessary energy consumption. Fig. 4 illustrates this situation of unreasonable traction when the train departures from the previous station; 
iii) Performance based regulation does not consider the passenger comfort with the vibrating speed profile. In the case of fig. 2, with the speed restrictions at the intersection, train will unavoidably accelerate to the high speed before it run into the low speed area. The dramatically change of the velocity with reduce the passenger ride comfort.

In this genetic algorithm, the optimization object can be set to the energy consumption, passenger comfort, or even both of them. In this paper, to simplicity the calculation of the fitness, the single-object fitness function, energy consumption was used for the calculation.

Fig. 3 is the civil situation of this simulation, in which the stations are located at 200 and 2600 in the chainage coordinates. Train head will start from point 200 and stop at point 2600. Together with the typical energy saving slope set in the civil situation, there were 2 speed restriction areas in this section to emphasize the optimization result.

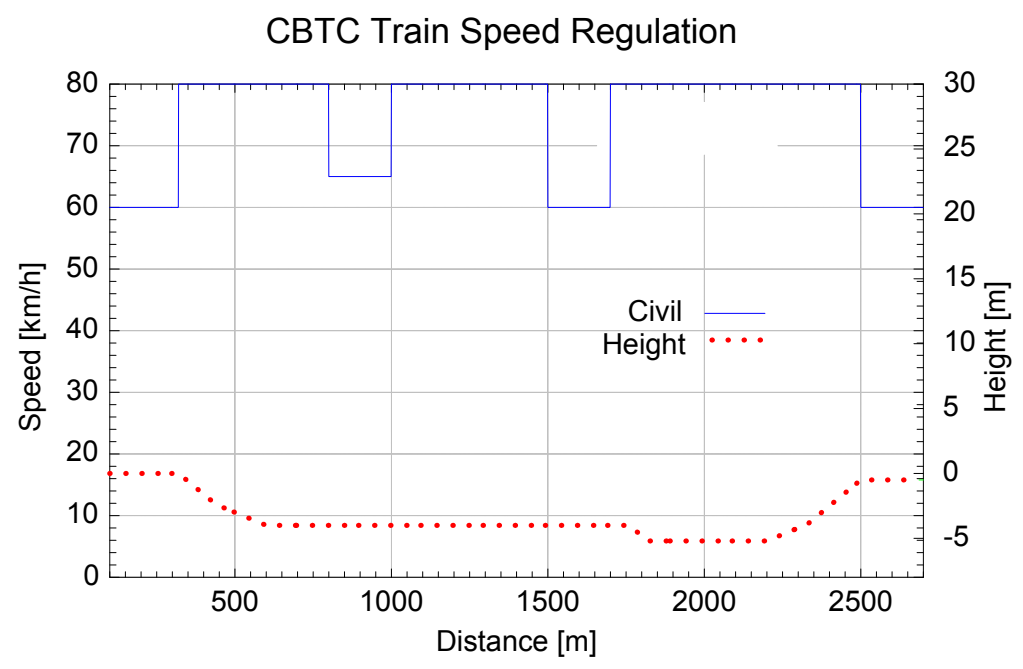

Figure 3: Grade and inter-station speed restriction.

Fig. 4 is the train actual speed profile in each performance level (PL2-PL4) and the corresponding energy consumption. In whatever the performance level selected, train had the unreasonable tracking and braking effort in the movement that cost the additional energy consumption, as well as reduced the passenger comfort. Since the PL1 is the most maximum speed profile, it cannot be optimized for reducing the energy consumption.

The object of the algorithm was to find the more optimized train actual speed profile between the 2 stations. 
CBTC Train Speed Regulation
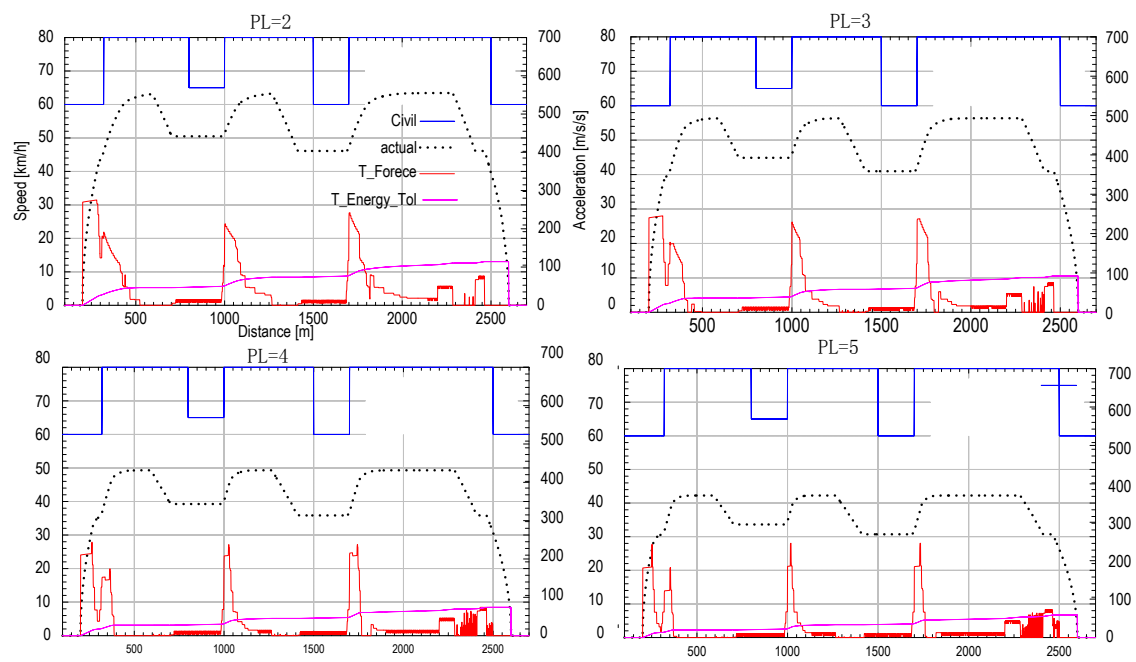

Figure 4: Simulation and energy consumption of each performance lever.

\section{The genetic algorithm based train speed regulation}

\subsection{Mathematical modelling}

The speed vector $\{\mathrm{x} 1, \ldots, \mathrm{xn}\}$ was applied to represent the command speed for the train movement in the section, in which $x i$ is the velocity at the speed change point. In this algorithm, the value of xi is from the velocity of PL1 to the velocity of PL5 at this point. Precision of the velocity value is set to $1 \mathrm{~km} / \mathrm{h}$. Command speed sample point in the 2400 meters long section is set to 50 in this simulation. All the command speed profile can be represent by

$$
x \in X=\left\{\begin{array}{cccc}
x_{11} & x_{12} & , \ldots, & x_{1 n} \\
x_{21} & x_{22} & , \ldots, & x_{2 n} \\
\ldots & \ldots & \ldots & \ldots \\
x_{m 1} & x_{m 2} & , \ldots, & x_{m n}
\end{array}\right\}
$$

The question can be abstracted as in the eqn (1), which command speed vector can instruct the train to move at the most optimized actual speed. Since the combination could be a very huge amount, the genetic algorithm (GA) is one of the most suitable methods for finding the best solution. 


\subsection{Implementation of GA}

GA is an effective optimizing technique that has been applied to various fields. The implementation of GA contains 6 steps: reproduction, crossover, mutation, gene duplications, gene deletions and reordering of genes within chromosome.

\subsubsection{Gene and chromosome}

Floating coding was used in this algorithm. A speed profile can be represented by:

$$
x_{i j} \in S_{j}=\left\{v_{j_{-} p l 5}, v_{j_{-} p l 5}+1, \ldots, v_{j_{-} p l 1}\right\}, j=1,2, . ., n
$$

where, $v_{j_{-} p l 5}$ is the velocity of point $\mathrm{j}$ in the PL5 speed profile, $v_{j_{-} p l 1}$ is the velocity of point $\mathrm{j}$ in the PL1 speed profile. $\mathrm{Sj}$ is the set of the float value can be selected at point $\mathrm{j}$ in the section. The amount of the set is depended on the velocity range between PL1 and PL5 at this point.

In the train speed regulation system, a chromosome of the genetic algorithm $x_{i}=\left(x_{i 1}, x_{i 2}, \ldots, x_{i k}, \ldots, x_{i n}\right)$ is a train command speed profile that the train will move according to. In eqn (1), $\mathrm{m}$ is number of the chromosomes. In the simulation of this paper, we can get a good simulation result when $\mathrm{m}$ is set to 200.

A genetic algorithm shall initialize a population of chromosomes. Each chromosome, i.e. an individual, is a possible solution to the optimization problem. The next generation population of chromosomes will be randomly selected from the current generation with selection probability based on the evaluation score of each chromosome. In eqn (1), $\mathrm{n}$ is the number of the genes. In the train speed regulation system, the number of genes depends on the number of the command speed change point. In the simulation of this paper, we can get a good simulation result when $\mathrm{n}$ is set from 20 to 50 .

\subsubsection{Genetic operation}

The individual $i$ can be initialized as:

$$
x_{i j}=\operatorname{rand}\left(S_{j}\right), j=1,2, \ldots, n
$$

where, $\operatorname{rand}\left(S_{j}\right)$ is the randomly generated from $S_{j}$, which is the set of possible train command speed value at position $\mathrm{j}$.

Once the initial population is built, the main operation of the genetic algorithm is to find a solution to the optimization problem using five basic steps to evolve the population from one generation to the next: selection, reproduction, crossover, mutation and fitness.

During each successive generation, some individual solutions are selected through the fitness-based process, which will be particularly described in the next section. Selection methods rate the fitness of each individual and select 
the best solutions to the next generation. Roulette wheel selection method was used in this paper. The reproduction probability of an individual is:

$$
P_{i}=\frac{\operatorname{Fit}\left(f\left(x_{i}\right)\right)}{\sum_{i=1}^{m} \operatorname{Fit}\left(f\left(x_{i}\right)\right)}, \quad i=1,2, \ldots, m
$$

where, $\operatorname{Fit}\left(f\left(x_{i}\right)\right)$ is the fitness of individual $x_{i}$.
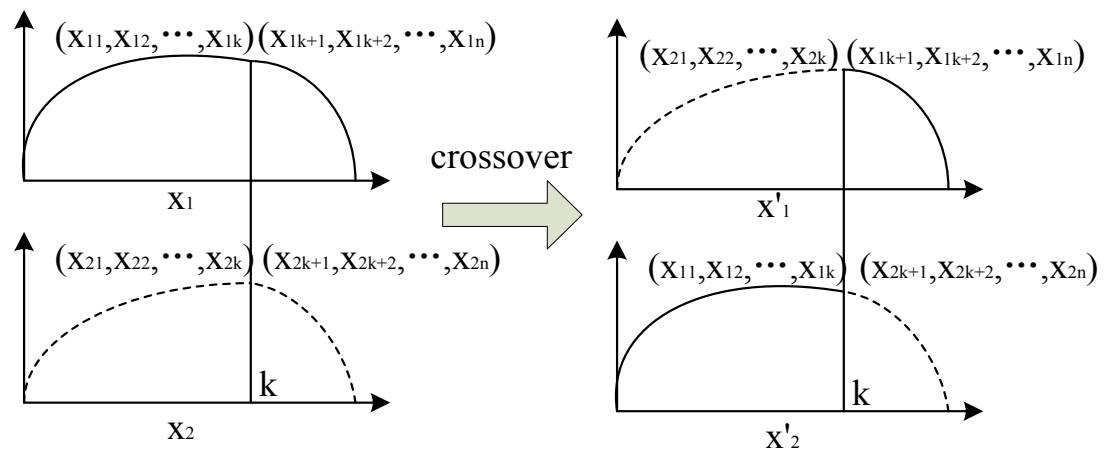

Figure 5: Speed profile crossover process.

After the individuals are selected, the reproduction will cross the individual's chromosomes to produce their offspring's chromosome. In the simple case, this involves exchanging genetic information by swapping bits within the parent's chromosome. Crossover is a random process. In the research of this paper the chromosomes are crossed at a single point. Two parents are selected for crossover at the defined crossover points to develop a new offspring, fig. 8. In this algorithm the crossover rate is $P_{c}=0.8$.

Mutation is used to maintain genetic diversity from one generation to the next. It alters one or more gene values in a chromosome from its initial state. After the mutation, a solution may be changed entirely from the previous one. If the Chromosome of individual $x_{i}$ is :

$$
x_{i}=\left(x_{i 1}, x_{i 2}, \ldots, x_{i k}, \ldots, x_{i n}\right)
$$

A random position of $x_{i}$ was replaced by the random value:

$$
\begin{aligned}
k & =\operatorname{rand}(n) \\
x_{i k}^{\prime} & =\operatorname{rand}\left(S_{k}\right)
\end{aligned}
$$

where, $S_{k}$ is the set of the permitted command speed for the position k. 
After the mutation a new individual is:

$$
x_{i}^{\prime}=\left(x_{i 1}, x_{i 2}, \ldots, x_{i k}^{\prime}, \ldots, x_{i n}\right)
$$

Mutation occurs during evolution according to a user-defined mutation probability. This generational process is repeated until a termination condition has been reached. In the research of this paper the mutation probability is set to 0.001 , and after 50 generations evolution the satisfied solution will always be found.

\subsubsection{Fitness functions}

A fitness function is a particular type of objective function that is used to summaries, as a single figure of merit, how close a given design solution is to achieving the set aims. If the energy consumption of the train moving along with the speed command profile is the object function $f(x)$, the fitness of individual $x_{i}$ is: others

$$
\operatorname{Fit}\left(f\left(x_{i}\right)\right)=\left\{\begin{array}{cc}
C_{\max }-f\left(x_{i}\right), & f\left(x_{i}\right)<C_{\max } \\
0, & \text { others }
\end{array}\right.
$$

where, $C_{\max }$ is a positive number.

The resistance and the track gradient must be taken into consideration in the $f(x)$ calculation.

3.2.3.1 Train resistance Train resistance is composed of basic resistance and the additional resistance [12].

$$
F_{\text {resistance }}=F_{\text {basicresistance }}+F_{\text {additionalresistance }}
$$

The basic resistance can be expressed as the Davis Formula:

$$
w_{o}(x)=A+B v(x)+C v^{2}(x)
$$

where, $v(x)$ is the train velocity at the position $\mathrm{x}$. A,B,C are the specified parameters in a project. In the research of this paper, $\mathrm{A}=1.08, \mathrm{~B}=0.008$, $\mathrm{C}=0.00096$.

In the urban rail transit system, the additional resistance $w_{d}$ is composed of the curve resistance $w_{r}$ and tunnel additional resistance $w_{s}$ :

$$
w_{d}(x)=w_{r}(x)+w_{s}(x)
$$


3.2.3.2 Track grade When the train moves on the slope, it experiences the component force of the gravity, if $w_{i}(x)$ is the unit grade resistance, then [12]

$$
F_{\text {grade }}(x)=w_{i}(x) M_{\text {train }} g
$$

3.2.3.3 Train traction force The actual traction force (net force) of the train at position $x, F_{n e t}(x)$ is:

$$
F_{n e t}(x)=M_{\text {train }}(1+\alpha) a(x)
$$

where, $\alpha$ is the train rotational rate, which is related to the train passenger load. In the research of this paper, passenger load is set to AW2, train rotational rate $\alpha=1.08$.

When the train is in the acceleration process, then $a(x)>0$. When the train is in the deceleration process, then $a(x)<0 . F_{n e t}(x)$, which is composed of train traction force, braking force, resistance and the gradient.

$$
\begin{aligned}
& F_{\text {net }}(x)=F_{\text {tracking }}(x)+F_{\text {braking }}(x) \\
& +F_{\text {resistance }}(x)+F_{\text {grade }}(x)
\end{aligned}
$$

3.2.3.4 Traction energy According to eqn (12) the train traction energy from $\mathrm{S} 1$ to $\mathrm{S} 2$ is:

$$
\begin{aligned}
& E_{\text {tractive }}=\int_{S 1}^{S 2} F_{\text {tractive_force }}(x) d x \\
& =\int_{S 1}^{S 2}\left(F_{n e t}(x)+F_{\text {resistance }}(x)+F_{\text {grade }}(x)\right) d x
\end{aligned}
$$

\section{Simulation}

\subsection{Civil information}

Table 1 is the civil information used in the research of this paper. Train departures from position 200 meter and stops at position 2600 meter. The energy saving grade was set in the simulation, fig. 3 . To study the efficiency of the genetic algorithm the more complicated speed restriction area were set in the simulation. 
Table 1: Civil information in the simulation.

\begin{tabular}{|c|c|c|c|}
\hline $\begin{array}{c}\text { Position } \\
(\mathrm{m})\end{array}$ & $\begin{array}{c}\text { Civil Speed } \\
(\mathrm{m} / \mathrm{s})\end{array}$ & Stop Point (stop $=1)$ & $\begin{array}{c}\text { Grade (\%, downhill is } \\
\text { negative) }\end{array}$ \\
\hline 100 & 60 & 0 & 0 \\
\hline 200 & 60 & 0 & -0.02 \\
\hline 320 & 80 & 0 & -0.01 \\
\hline 440 & 80 & 0 & 0 \\
\hline 600 & 80 & 0 & 0 \\
\hline 800 & 65 & 0 & 0 \\
\hline 1000 & 80 & 0 & 0 \\
\hline 1500 & 60 & 0 & 0 \\
\hline 1700 & 80 & 0 & -0.02 \\
\hline 1760 & 80 & 0 & 0 \\
\hline 1760 & 80 & 0 & 0.01 \\
\hline 1820 & 80 & 0 & 0.02 \\
\hline 2200 & 80 & 0 & 0 \\
\hline 2330 & 80 & 0 & 0 \\
\hline 2500 & 60 & 1 & 0 \\
\hline 2600 & 80 & 0 & \\
\hline 3300 & 80 & & 0 \\
\hline
\end{tabular}

\subsection{Simulation result}

The following are some typical speed profiles generated in the simulation.

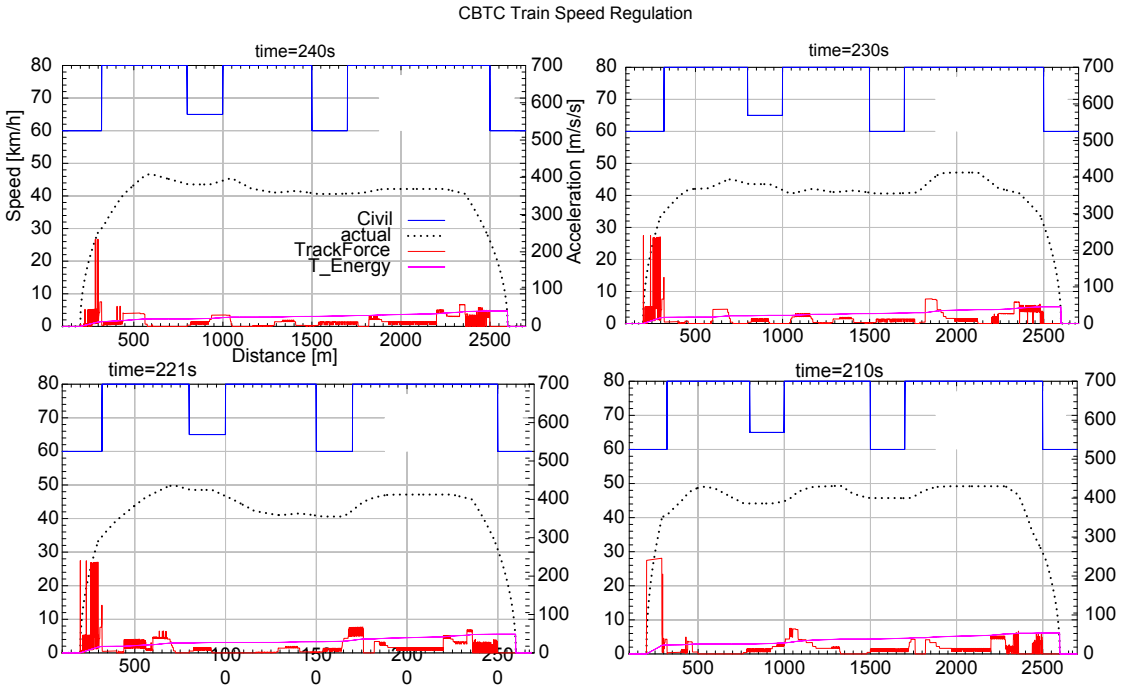

Figure 6: Speed profiles. 
Based on the genetic algorithm the train operation during the inter-station can be greatly optimized for the energy consumption, as well as the passenger ride comfort for the consideration of reducing the dramatic braking and tracking frequency for each time precision speed profile.

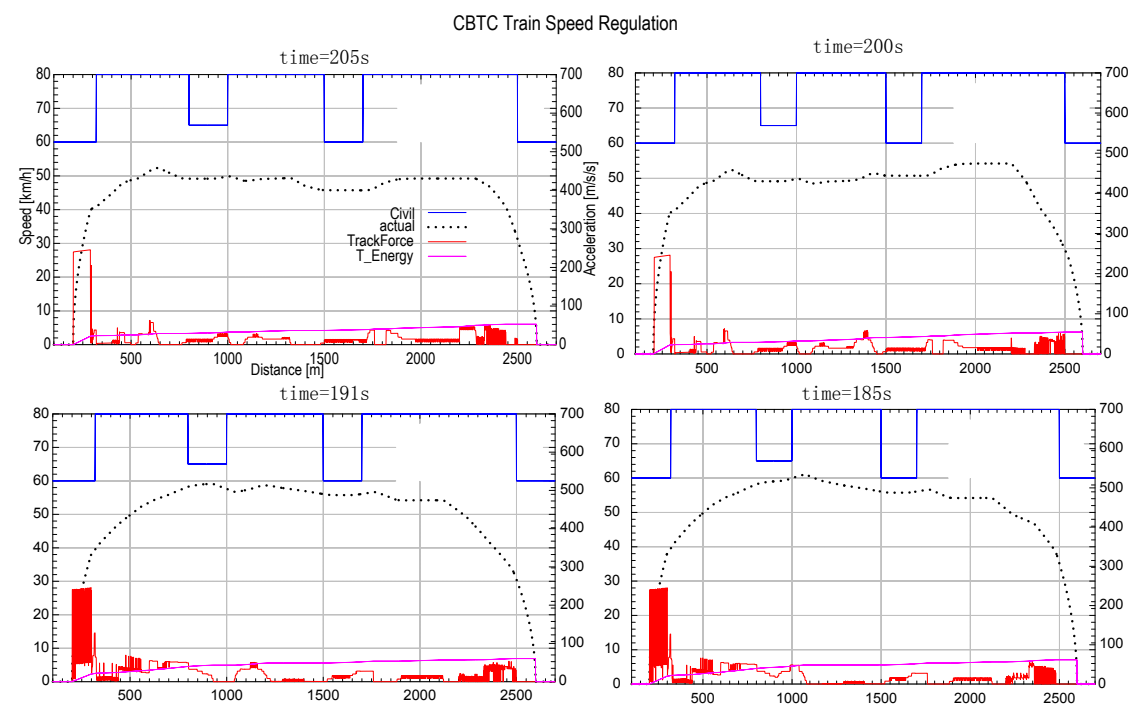

Figure 7: Speed profiles.

\section{Conclusion}

Compared to the traditional performance levels command which were generated by the ATS subsystem, the enhanced genetic algorithm based train speed regulation algorithm can object to saving the energy consumption, improve the passenger travel comfort at each defined inter-section travel time. In this algorithm, each travel time target speed can be reflected by its particular speed profile which has been optimized by the genetic algorithm with the multi-objects of energy and passenger comfort.

\section{References}

[1] Xun Jing, Ning Bin, Gao Chunhai. Study and realization of train tracking simulation system [J]. Journal of Beijing Jiaotong University, 31(2), pp. 34-37, 2007.

[2] Chen Rongwu, Guo Jin. Development of the new CBTC system simulation and performance analysis [C] // NING Bin. Proceedings of 12th international conference on computer system design and operation in railways and other transit systems. Beijing: WIT Press, pp. 497-507. 
[3] Xue Yanbing, Ma Dawei, Wang Lie. Calculation method of energy consumption in train traction [J], China Railway Science, 28(3), pp. 84-87, 2007.

[4] Ke B. R., Chen N. Signalling blocklayout and strategy of train operation for saving energy in mass rapid transit systems [J]. IEE Proc. -Electr. Power Appl., 152(2), pp. 129-140, 2005.

[5] Francois Ruelland, Kamal Al-Haddad. Reducing subway's energy [C] // Proceedings of 2007 IEEE Canada Electrical Power Conference. Canada: IEEE Press, pp. 261-267, 2007.

[6] Yu Jin. Study on multi-objective optimization of train operation process and its control strategies [D]. Chengdu: Southwest Jiaotong University (in Chinese), 2007.

[7] Shi Guohong. Study on train operation and movement process simulation and its' optimization [D]. Chengdu: Southwest Jiaotong University, (in Chinese), 2006.

[8] Lu Fei. Operation optimization of the subway train under moving block system [D]. Shandong: Shandong University, (in Chinese), 2006.

[9] Yu Jin, He Zhengyou, Qian Qingquan. Multi-objective train operation optimization based on particle swarm algorithm [J]. Journal of Southwest Jiaotong University, 45(1), pp. 71-75, 2010.

[10] Li Yusheng, Hou Zhongsheng. Study on energy-saving control for train based on genetic algorithm [J]. Journal of System Simulation, 19(2), pp. 384-387, 2007.

[11] Li Bo. Study on energy-saving control for train based on genetic algorithm [D]. Chengdu: Southwest Jiaotong University, 2007, (in Chinese).

[12] Chen Rongwu, Zhu Changqian, Liu Li. CBTC based urban rail transit train energy consumption algorithm and simulation [J]. Application Research of Computers, 2011, 28(6), pp. 2126-2129.

[13] Chen Rongwu, Liu Li. Optimization algorithm of train operation energy consumption based on genetic algorithm [J], Journal of Traffic and Transportation Engineering, 12(1), pp. 108-114, 2012. 